\title{
Value Analysis on Purely Decorative-Type Digital Animation
}

\author{
Wangqiao Rong1,2 \\ ${ }^{1}$ School of Fine Arts, Nanjing Normal University, Nanjing, China \\ ${ }^{2}$ Jiangsu Center for Collaborative Innovation in Geographical Information Resource Development and Application, Nanjing, China \\ Email: 1062643722@qq.com
}

How to cite this paper: Rong, W. Q. (2018). Value Analysis on Purely Decorative-Type Digital Animation. Art and Design Review, 6, 61-70.

https://doi.org/10.4236/adr.2018.62006

Received: April 12, 2018

Accepted: May 15, 2018

Published: May 18, 2018

Copyright (c) 2018 by author and Scientific Research Publishing Inc. This work is licensed under the Creative Commons Attribution International License (CC BY 4.0).

http://creativecommons.org/licenses/by/4.0/

\begin{abstract}
Purely decorative-type animation has its own living space in the field of animation. It has unique practicality and aesthetic value in decorative applications. Purely aesthetic pursuit for form, constantly changing visual presentation in the interaction and non-interaction, convenient editing and re-editing of images, online sharing of decorative animated resources can be realized off-site, real-time maintenance and updating and information dissemination capabilities, which highlights its value lies. Diversified methods of production in turn provide a guarantee for the realization of its value and provide the possibility of extending its value.
\end{abstract}

\section{Keywords}

Digital Animation, Decoration, Value, Programming, Interaction

\section{Introduction}

Digital animation is generated by the computer and spread in digital format, has a unique artistic appeal and a very wide range of applications, and has exceeded the people's function cognition which is inertial and stayed in the traditional film and television animation. Digital animation can be applied to almost every corner of real life. As one of the branches, the value realization of purely decorative-type digital animation has more realistic meanings. This article tries to discuss from the aspects of logical reasoning, feature analysis and generating methods.

\section{Logical Reasoning Means That There Is Necessity}

\subsection{Digital Animation and Digital Painting}

Digital animation refers to the use of graphics and image processing techniques, 
with the aid of programming or animation software to generate a series of scene images, where the current frame is a partial modification of the previous frame. Computer animation is the effect of using a method of continuously playing a still image to produce the motion of an object (Parent, 2012). Earlier, these images were entirely hand-painted by hand and are now heavily or even completely computer-generated, so the relationship between animation and painting is very approaching. Drawing is the basis of animations. To some extent, digital animation is an extension of painting function to mankind under the condition of highly developed science and technology, especially movie technology and computer graphics and image technology. However, animations are not just paintings, but a combination of visual and auditory. Depending on the image presentation, the animation can also be used to achieve some kind of atmosphere rendering or special practical functions.

Because of the application of digital technology, animation making is much easier than manual. Nowadays, animations have evolved from only a small number of people engaged in the field at the very beginning to immeasurable people favorites today. At the beginning animations was primarily just for entertainment, publicity, now have developed entertaining, gaming, interacting, displaying, roaming and augmented reality, teaching, scene reproduction, decoration and other functions in one (Huang, 2005).

\subsection{Decorative Painting and Decorative-Type Digital Animation}

Decorative painting is a branch of painting, its decorative function being purified, and the fundamental difference from the general painting is not in the theme and content, not in the tools and means, but in the form of expression. In other words, the essence of this difference is the different sense of formality. The greatest strength of decorative art lies in "diversification". The decorative painting itself can be an independent type of painting, which is evolved from a variety of paintings "changing". Decorative painting is the inevitable result of the development of painting art. Even Chinese literati painting, its content, form and painting purpose, also have obvious decorative elements, closely connected with the decorative art and influence each other (Gombrich, 2000).

Decorative painting is bound to breed decorative-type animation. In the past, it was obviously extravagant or very risky to use animations only for decoration, as images were not easy to generate. Nowadays, computers are used to quickly obtain high-quality graphic images that can meet various presentation needs. In particular, it is a specialty of computers to generate graphic images with the features of mathematical models. As long as the idea is smart, it is entirely possible and sometimes even very easy to get a rich charming decoration animation. It is precisely because the computer has been involved in the entire process of animation generation process, so the current decoration animation has already been purely decorative-type digital animation. Pure decorative-type animation refers to an animation type that is purely used for decoration, using digital technology. 
It is a combination of decorative design art and digital animation technology (Figure 1).

\subsection{Decoration and Digital Decoration}

Mankind has been working to tirelessly pursue beauty. Whenever a new material, technology created, people will try to beautify their life with them. The modern world culture has undergone a focus shift from the oral culture to typography culture and then to image culture. When digital images are presented in their fantastic, omnipotent virtual appearance to the world, people are convinced that digital image decoration must also be indispensable, since the decoration has to be existing. The goal of digital decoration and traditional decoration can completely be consistent, that is, can be used to beautify the environment and harmonious mood. However, digital technology has created a virtual world paralleled to the real world which requires a lot of decoration, and can only rely on digital decoration. For example, various web interface, game software scene, music visualization, and so on (Wang, 2005).

The way of digital decoration also inherits the traditional decoration, for instance, with the help of the form elements: point, line and surface using the form principles: change and unity, contrast and harmony, rhythm and cadence, symmetry and equilibrium, and so on to create an effect of decorative beauty. However, the digital expression and the application scope are more flexible and broad, which is an emerging field of modern decoration and also an unprecedented development in the field of decoration (Dunn, \& Parberry, 2005).

Digital decoration takes digital content as the core, from the shape and structure to distinguish, which can be decorative-type digital painting, and can also be decorative-type digital animation; which can be two-dimensional, and can be three-dimensional; which can be a regular shape, it can be a special shape.

Decorative-type digital animation has been increasingly used for decoration because of its many advantages.

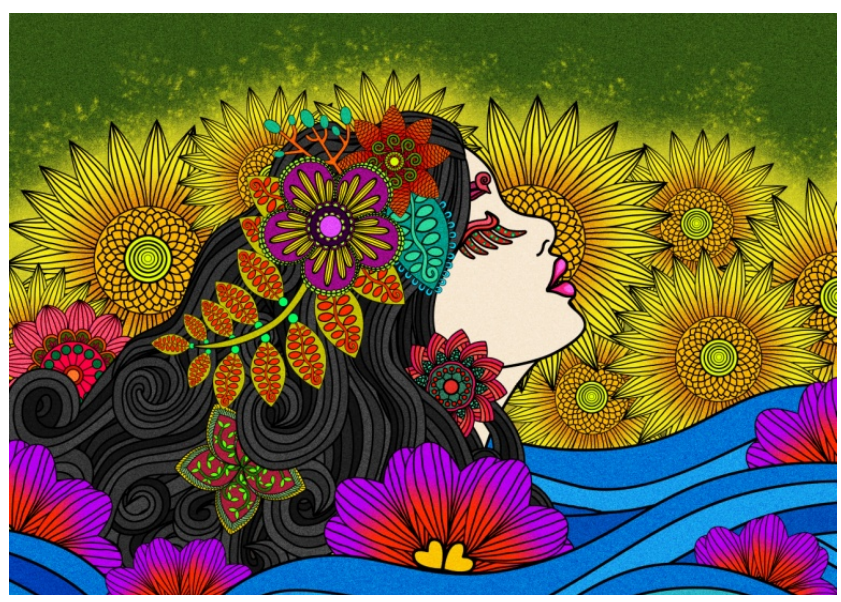

Figure 1. Decorative-type digital animation "Flower Dance" (Author: Yawen Zheng). 
Decorative-type digital animation also has the distinction of two-dimensional and three-dimensional. Two-dimensional animation can convey the sense of plane painting, which can give full artistic appeal caused by the change of form. The three-dimensional animation can vividly simulate the real or imaginary objects and can make every effort to highlight the sense of space and the lens so as to give audience visual shock. With the continuous improvement and popularization of modern three-dimensional animation, especially the 3D animation technology with stronger depth perception and the diversification of display technologies, the decoration demand of the people for digital animation will be more and more urgent, especially display media becoming thinner and softer and the process rapidly advancing. It is particularly worth mentioning that it is based on the imaging principle by photo-chromic to have dynamic visual communication, through the electronic screen or projection device presented, so the brightness of dynamic decorative images can be controlled in logical and physical two ways to achieve a specific space lighting and segmentation (Boler, Mandyberg, \& Zhang, 2010).

\section{The Features Highlight and Determine the Value of Existence}

\subsection{Cute Because of "Simple"}

From the creativity point of view, the difference between decorative-type animation and video animation, game animation and other types of animation is that the goal is very simple. That is to achieve the formal beauty, simple beauty and the order beauty so as to convey the pleasure for the audience enjoyed. The focus of creativity lies in the form composition, generally do not pursue the storyline, checkpoint definition, just a combination of digital patterns, objects or notes, in order to have the sense of form and the sense of rhythm. Its characteristics are simple, plane, space-time and order. As a result of this purely form aesthetic pursuit, the decorative aesthetic value of decorative-type animations is particularly prominent, more and more "cute" and more frequently used for decoration (Gombrich, 2001).

\subsection{Attract People's Attention Because of "Vivid"}

The visual experience of decorative art as a space art is not just static. Many times in reality may be dynamic. For example, viewing the large mural of the China Millennium Monument, "The Song of China," the physical body and viewpoint need to make substantial movements to see the whole story, which includes the continuity of time here. Digital animation is a typical complex of space and time together in one. Display area of the digital animation can be large or small, and for small one it maybe needn't turn the body, the whole panorama can be seen. People can feel these ever-changing decorative images with a strong sense of forms in relaxed. In public places, digital animation can be reproduced with a large-size display screen or projection device, such as the canopy image 
decoration in the Old Town of Las Vegas of the United States, which not only has a large scale, but also needs to be uplifted for a kilometer to look up. And the decorative image itself is also dynamic, depended on multiple projectors mapped to the canopy, and the patterns edited by the computer change almost daily. This new means of decoration, the synthesis of sound, light, electricity and other modern technology, can bring great shock to the audio and visual senses, meanwhile the dimension of aesthetic-power fields which produces is multidimensional.

The needs of digital decoration generate digital decoration animation. If just relying on digital static images as a decorative means, its superiority can't be prominent. The traditional visual sculpt arts, such as decorative painting, can be used to replace. If the decorative-type digital animation is introduced into the field, the advantages of digital decoration will immediately be demonstrated.

Digital animation belongs to the category of digital media art, and it has the characteristics of popularization of digitalized media art (Li, 2006). Any art that can become popular must be an art that has the needs of the public experience and appreciation, and is easy to communicate with the public soul and achieve emotional resonance. Decorative-type digital animation will inevitably become a popular decoration art. The most important reason is that it can "move and change". In some extent people's pursuit for "moving and changing" is out of the instinct as a human being because life itself is constantly changing and moving, while this life characteristic has formed a corresponding psychological demand hint of visual art experience for "vividness" in the living body. Therefore, digital animation can also play the full role to its features of "moving and changing" in addition to the general digital decorative performance. For example, Figure 2 shows a stage on the background of digital animation. The digital animation is purely for the sake of beautifying the environment and enhancing the atmosphere. Because it is in an uninterrupted cyclical changing, it can even be induced by interaction with the actors. The audiovisual experience feeling is very rich and intense, so it can particularly stimulate the audience's interest. This is incomparable to the common static decorative background in the past.

There are two types of "moving and changing" in decorative-type digital animation. One is active, that is autonomously circulatorily presented in strict accordance with the content and sequence of the initial definition, and its process needn't be interfered or redefined by the outsides. Under normal circumstances, the results of each cycle process are the same. However, sometimes it can be random, that is, the pictures or the sequence in each cycle process are different. The other is passive, that is, the presentation of the pictures (including static and dynamic) is conditional and requires the aid of the external "interference", otherwise the pictures will not appear or remain unchanged; in addition, the result of the picture changes will differ from each other because of the "interfered" difference. This "interference" is actually the so-called interactive experience process (Figure 3 ). 


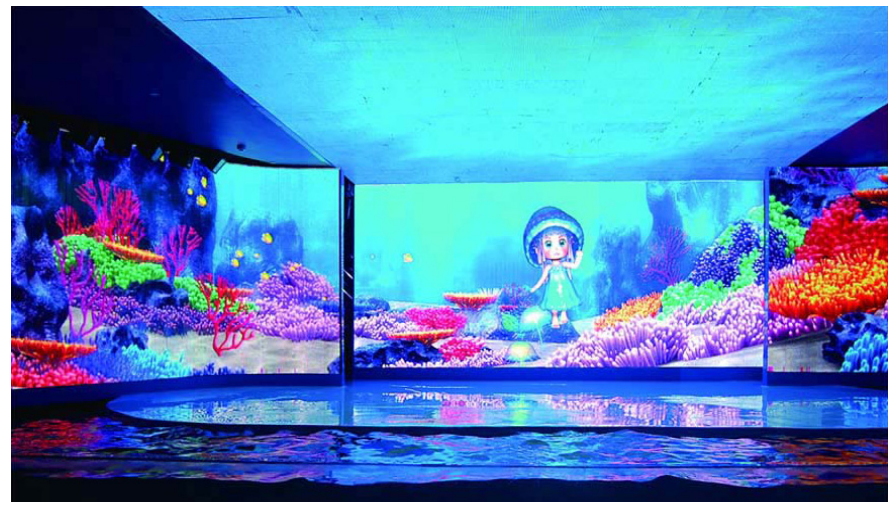

Figure 2. Choreography visual creativity using decorative-type digital animation (Source: http://www.cgtiger.com).

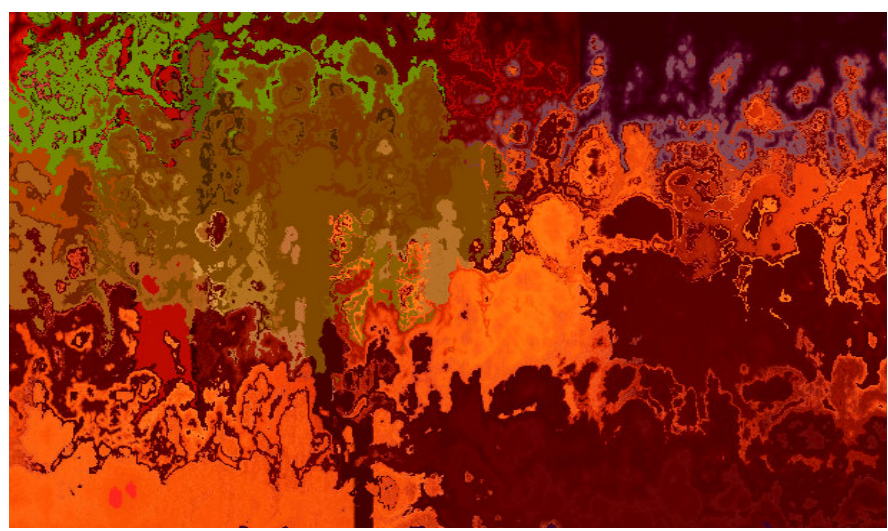

Figure 3. Decoration animation with interactive sensing function (Author: Wangqiao Rong).

Decorative-type digital animations with interactive experience effects have more space for decoration applications. From an aesthetic point of view, because of the interaction between the subject and object of aesthetics, it is easy to mobilize the curiosity and intimacy of the subject of aesthetics, thus the aesthetic behavior is easy to reach and the aesthetic perception of the aesthetic object can be conveyed more frequently and effectively. From a practical point of view, because of the real-time and pertinence of interaction, we can combine the daily practical needs of lighting, temperature, sound adjustment, safety tips, service response and other in decorative environment with the decorative animation skillfully in order to achieve the value of intelligent grafting and peripheral derivatives extending (Grigg, \& Zambado, 2003).

\subsection{Be Favored Because of "Changeable"}

First of all, decorative-type digital animation picture is in constant change, and this change is to strictly follow the dynamic visual art performance rules, so it can accurately convey the form aesthetic feeling of decoration. In addition, decorative-type digital animation has enough room to change, whether it is interactive or non-interactive type. To change is more convenient, and it will not add 
too much cost. If it is a decorative animation programmatically generated, then it's more convenient: just change the relevant parameter values to get a new visual effect (Figure 4). For interactive types of decoration animations, "changeable" has two meanings, one is the variability of content generation, which is similar to the variability of the non-interactive decorative-type animations described above. The other is the variability in content presentation, which is the animation content will be different with the results of human-computer interaction (Cooper, Reinmann, \& Cronin, 2008). For example, changing the relevant parameters in the decorating animation program of Figure 4 (a) can change the shape of the flower in real time (Figure 4(b)) and the number of petals (Figure $4(\mathrm{c})$ ), adjust the speed at which the drop falls (Figure $4(\mathrm{~d})$ ), and so on.

It is precisely because of the "changeable" characteristics in the implementation of digital animation decoration function. And it can be used to the maximum extent to meet people's complex needs for environmental decoration, so it has been more and more popular, especially the "changeable" in the second layer of interactive-type decoration animation seems even more so.

\subsection{Reluctant to Be Apart Because of "Networking"}

The decorative-type digital animation is an emerging form of decorative arts, and at the same time, it belongs to digital media art. Decorative art and digital media art both have the characteristics of "attachment" and "neutrality". Their existence and development cannot avoid the social status of science and technology and the status of economic development. The rapid development of the Internet, especially the broadband Internet, has made it possible to transmit digitized works of art remotely, so the decorating application of decorative-type digital animation is bound to be "networked".

Network can achieve decorative-type animation resource sharing in off-sites, and realize network management. Decorative content can achieve constantly real-time maintenance and updating. Network also allows decorative-type digital animation to realize the conversion from image to information or directly to implant a variety of information to implement the function of information dissemination, which can make animation not only be an important decorating means in the living environment of human society, but also be an indispensable medium in life and work.

The above networked benefits are long-lasting, widespread, and additional "bonuses" from digital animation decorations that continuously stimulate the decorating demand for decorative-type digital animation. In a sense, this also promotes its value.

\section{Generation in "the Right Way" to Ensure the Realization of Value}

If something more valuable is not easy to come by, it will never be seen or used by the world, and its value will not be realized and be proved. It will eventually 


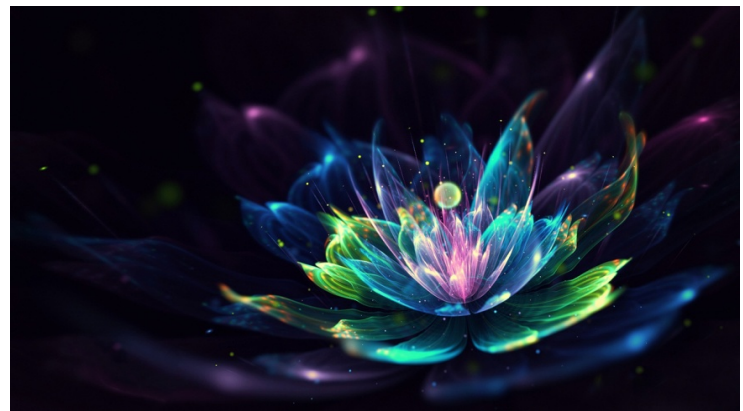

(a)

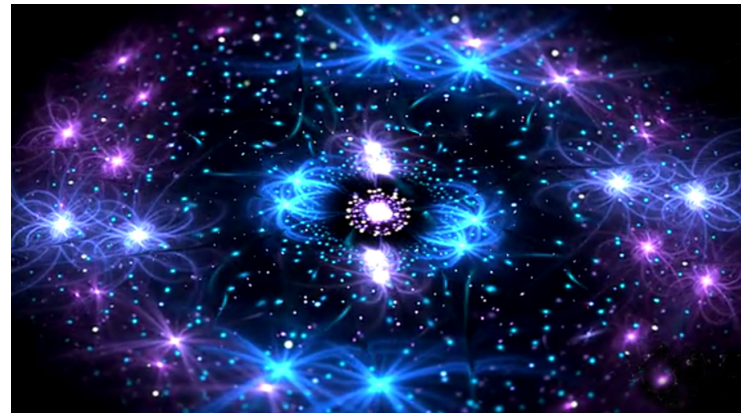

(b)

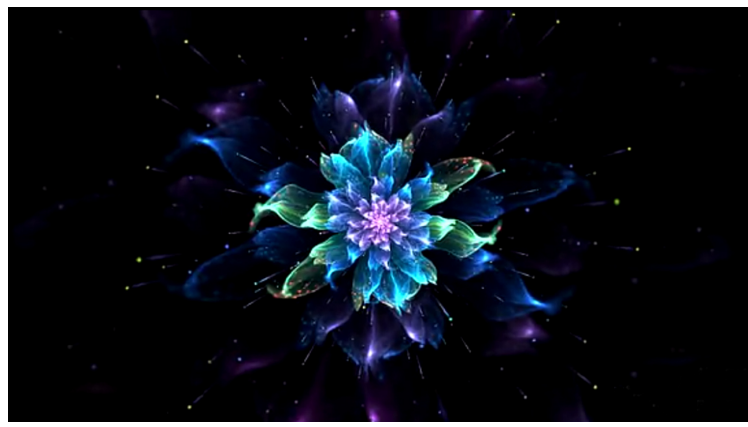

(c)

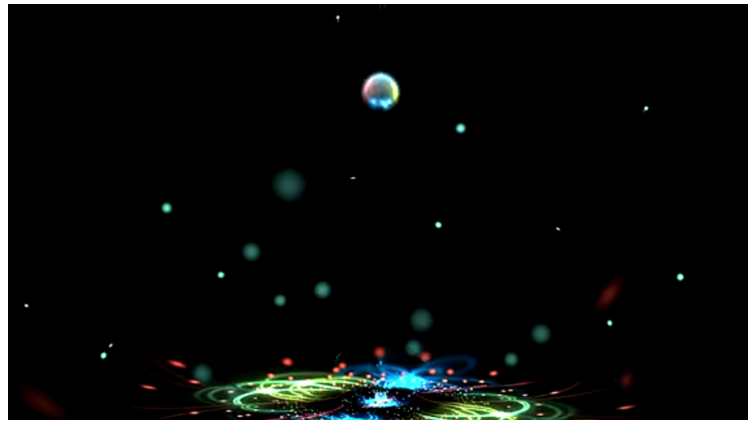

(d)

Figure 4. (a) Based on Fractal Algorithm Programming to Create the Animation Illusion Flower from water points (Author: Chen Lin); (b) changing parameters obtained different shapes flowers (Author: Chen Lin); (c) changing the parameters gets a different number of flowers (Author: Chen Lin); (d). changing parameters real-time change of water droplets falling speed (Author: Chen Lin). 
be abandoned by the world and forgotten, thus losing the existence meaning of value. The reason why decoration-type digital animation has extraordinary value is that it has broad prospects for development not only because of its superior characteristics but also because of its diversified generating methods such as drawing support from interactive operating platform, algorithm-based programming, interaction operation and coding programming combination, etc., can be expected and accessible.

Continuously updating digital image software and hardware technologies make dynamic image generation not only easier and more splendid, therefore whatever the quantity or the quality both provide possibility for decorative applications. Cheap display media and platforms with high-definition image have been launched for the dissemination of high-end digital animation providing a solid material foundation. Digital storage space has become more and more, and even the latest cloud computing makes storage space no longer a concern for users, which provides great convenience for the mass management of digital animation resources (Chen, Wang, \& Jin, 2009).

In general, the generation of purely decorative-type digital animation is easier than movie animation and game animation, but this will definitely not devalue its artistic and practical values, on the contrary, it is precisely because of its short production cycle, the low developing cost, less difficulty in maintenance, and larger freedom to subsequently upgrade, and other advantages existence that makes it very likely brewing and forming a sufficiently large scale market, coming into the tens of thousands of households.

\section{Conclusion}

Purely decorative-type digital animation has its own existing value, and its value space is very large. It is necessary to separate it from many types of digital animations to conduct professional exploration and research, in order to make it more in-depth, systematic, richer presenting, and more audio-visual aesthetic artistic effects, thus, will have more people be able to feel its fascinations. This will provide professional technical guarantees for the popularization of purely decorative-type animation, and provide the possibility for maximizing its value. It will also cultivate a new ecological environment for digital animation prospects and open up new and broad careers for digital animation artists.

\section{References}

Boler, E., Mandyberg, M., \& Zhang, J. (2010). Digital Art Design Foundation (p. 67). Beijing: People's Posts and Telecommunications Press.

Chen, Y., Wang, Q. B., \& Jin, Y. (2009). Virtualization and Cloud Computing (p. 53). Beijing: Electronic Industry Press.

Cooper, A., Reinmann, R., \& Cronin, D. (2008). About Face3 Interaction Design Essence (p. 83). Translated by Liu Songtao. Beijing: Publishing House of Electronics Industry.

Dunn, F., \& Parberry, I. (2005). 3D Mathematical Fundamentals: Graphics and Game Development (p. 98). Translated by Shi Yinxue, Chen Hong, Wang Rongjing. Beijing: 
Tsinghua University Press.

Gombrich (2000). The Sense of Order-The Psychological Research of Decorative Arts (p. 65). Translated by Fan Jingzhong. Changsha: Hunan Science and Technology Press.

Gombrich (2001). Art and illusion-Psychological Research on Image Reproduction (p. 55). Translated by Lin Xi, Li Benzheng, Fan Jingzhong. Changsha: Hunan Science and Technology Press.

Grigg, R., \& Zambado, P. (2003). Psychology and Life (p. 94). Translated by Wang Lei, Wang Su. Beijing: People's Posts and Telecommunications Press.

Huang, M. F. (2005). Pan-Animation of 100 Ideas (p. 4). Xiamen: Xiamen University Press.

Li, S. D. (2006). Introduction to Digital Media Art (p. 44). Beijing: Tsinghua University Press.

Parent, R. (2012). Computer Animation Algorithms and Techniques, Second Edition (p. 36). Translated by Liu Yi. Beijing: Tsinghua University Press.

Wang, L. Z. (2005). Visual Art Psychology_Visual Effects and Psychological Analysis of Fine Arts (p. 47). Beijing: People's Fine Arts Publishing House. 